\title{
How are encyclopedias cited in academic research? Wikipedia, Britannica, Baidu Baike, and Scholarpedia
}

\author{
Xuemei Li; Mike Thelwall; Ehsan Mohammadi
}

How to cite this article:

Li, Xuemei; Thelwall, Mike; Mohammadi, Ehsan (2021). “How are encyclopedias cited in academic research? Wikipedia, Britannica, Baidu Baike, and Scholarpedia". Profesional de la información, v. 30, n. 5, e300508.

Manuscript received on $11^{\text {th }}$ June 2020 Accepted on $02^{\text {th }}$ August 2021

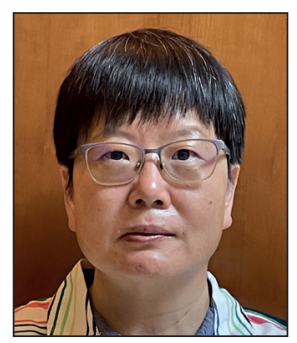

Xuemei Li $ه$

https://orcid.org/0000-0002-3384-1987

York University Libraries

Open Scholarship Department

4700 Keel Street, Toronto, Ontario,

Canada M3J $1 \mathrm{P} 3$

lixuemei@yorku.ca

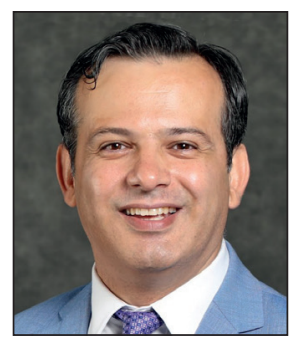

Ehsan Mohammadi

https://orcid.org/0000-0003-3481-6991

University of South Carolina

College of Information and Communications

1501 Greene Street, Columbia

SC 29208, USA

ehsan2@mailbox.sc.edu

\begin{abstract}
Encyclopedias are sometimes cited by scholarly publications, despite concerns about their credibility as sources for academic information. This study investigates trends from 2002 to 2020 in citing two crowdsourced and two expert-based encyclopedias to investigate whether they fit differently into the research landscape: Wikipedia, Britannica, Baidu Baike, and Scholarpedia. This is the first systematic comparison of the uptake of four major encyclopedias within academic research. Scopus searches were used to count the number of documents citing the four encyclopedias in each year. Wikipedia was by far the most cited encyclopedia, with up to $1 \%$ of Scopus documents citing it in Computer Science. Citations to Wikipedia increased exponentially until 2010, then slowed down and started to decrease. Both the Britannica and Scholarpedia citation rates were increasing in 2020, however. Disciplinary and national differences include Britannica being popular in Arts and Humanities, Scholarpedia in Neuroscience, and Baidu Baike in Chinese-speaking countries/ territories. The results confirm that encyclopedias have minor value for academic research, often for background and definitions, with the most suitable one varying between fields and countries, and with the first evidence that the popularity of crowdsourced encyclopedias may be waning.
\end{abstract}

\section{Keywords}

Wikipedia; Britannica; Baidu Baike; Scholarpedia; Encyclopedias; Scopus; Citation analysis; Open access; Scholarly communication; Multidisciplinary; Countries. 


\section{Introduction}

Crowdsourced encyclopedias are sometimes cited in academic research. This practice can be controversial because, unlike traditional scholarly encyclopedias (e.g., Tomaszewski, 2018), crowdsourced sites such as Wikipedia are publicly editable and dynamic and so do not provide the permanence and authority usually required for references in academic publications. Other encyclopedias may also not be subject to full academic rigor, even if subject to editorial review and written by invited experts. Nevertheless, since Wikipedia and other major encyclopedias seem to be usually accurate, it is arguably reasonable to cite them to guide article readers to useful background reading that does not underpin the logic of an article. There is no recent information about whether citations to Wikipedia or other encyclopedias are increasing, however, and whether they are drawn upon in different ways. This information is needed to help librarians, authors, and referees to understand the research contribution that a major encyclopedia can make, if any.

Wikipedia has become popular for both education and research since it started in 2001 (Kousha; Thelwall, 2017; Mesgari et al., 2015; Okoli et al., 2014), although the authority of its content has been repeatedly scrutinized for accuracy and coverage (Chesney, 2006; Giles, 2005; Holman-Rector, 2008; Jullien, 2012; Messner; DiStaso, 2013; Okoli et al., 2012; Samoilenko; Yasseri, 2014). One advantage of Wikipedia is its ability to react quickly to new issues, such as Covid-19 (Colavizza, 2020). Since Wikipedia summarizes knowledge for a general audience, often supported by references, it has been used as evidence of the wider impact of academic research to complement traditional citation-based indicators (Jemielniak; Masukume; Wilamowski, 2019; Kousha; Thelwall, 2017; Lin; Fenner, 2014; Priem; Piwowar; Hemminger, 2012). Wikipedia is usually cited to provide general information or a definition (Tohidinasab; Jamali, 2013). While citations from Wikipedia to academic publications may reflect knowledge transfer from academia to a wider public domain, or can be used to verify its information, citations in the reverse direction from academic publications to Wikipedia are more controversial (Fallis, 2008). Despite this, the number of academic citations to Wikipedia increased annually until at least 2015, including from reputable publications and traditional non-OA articles (Tomaszewski; MacDonald, 2018).

A few studies have investigated citations to Wikipedia from academic publications, starting with an investigation of the quality of Wikipedia articles that had been cited in online news stories (Lih, 2004). Some research has had a subject focus, investigating Wikipedia citations from law reviews (Baker, 2011; Shoyama, 2014), chemistry journals from three major publishers (Brazzeal, 2011), and health science journals in Medline, PubMed, or Embase (Bould et al., 2014). These studies have found that citations to Wikipedia were increasing over time, but there is disagreement over whether it is reliable and whether it should be cited by prestigious journals, including Nature, Science, and the BMJ. Both academic publications about Wikipedia and citations to Wikipedia in general increased over time shortly after its appearance (Huggett, 2012; Park, 2011), although it is not known if this has increased in the last decade.

In contrast to Wikipedia, Encyclopedia Britannica is a well-known, centuries-old English-language encyclopedia that seems to have a reputation for scholarly authority. Scholarpedia, a peer-reviewed free online encyclopedia that started in 2006 (Izhikevich, 2006), was the closest rival to Wikipedia in 2011 but received only 1/20 as many citations (Huggett, 2012). Baidu Baike is the most popular online Chinese general encyclopedia. It started in 2006 and a few studies have investigated citations from Chinese academic articles to it using the Chinese article index databases CNKI (2019) or CSSCl (Chinese Social Sciences Citation Index, 2015) (Ding; Zhang; Liu, 2013; Wang, 2016). Nevertheless, no studies have investigated how Baidu Baike is cited in Scopus-indexed articles.

This study compares citations from Scopus-indexed documents to Wikipedia, Britannica, Baidu Baike and Scholarpedia, with the objective of assessing whether changes over time and differences between them can give insights into the different roles that encyclopedias can play in academic research. Whilst there are many other encyclopedias that could have been included, these four have differences that may be illuminating. Wikipedia and Baidu Baike are crowdsourced encyclopedias. Whilst Wikipedia is multilingual, Baidu Baike is in Chinese. Britannica is an English peer-reviewed proprietary general encyclopedia with some content freely available online. Scholarpedia is an English peer-reviewed free online encyclopedia with substantial coverage of Astrophysics, Celestial mechanics, Computational neuroscience, Computational intelligence, Dynamical systems, Physics and Somatosensory systems. This article compares the numbers of citing documents to these four encyclopedias, breaking down the results by subject, language, publication type and author country.

\section{Research questions}

This project assesses trends in the uptake of four major encyclopedias in formal scholarly communication, and whether their uses are affected by characteristics of the citing documents and their authors. The following questions drive the investigation.

- Is the level of academic citing of Wikipedia, Britannica, Baidu Baike and Scholarpedia increasing over time?

- Which fields most cite the four encyclopedias?

- Are there differences between encyclopedias in citing document subjects, types (e.g., open access, journal articles, or books), publication languages, or author characteristics (e.g., national affiliations)? 


\section{Methods}

The evidence used to address the above questions is taken from explicit mentions of the four encyclopedias in academic literature reference lists. Scopus was chosen to count how many documents cite the four encyclopedias because Scopus covers more publications than does the Web of Science (WoS) and also it allows more comprehensive searches within the cited reference fields (Kousha; Thelwall; Abdoli, 2012; Li; Thelwall; Kousha, 2015).

Since Wikipedia was launched in 2001, citations to Wikipedia and Britannica were counted from 2002 to 2020. Both Baidu Baike and Scholarpedia were launched in 2006 and so citations to these two encyclopedias were counted from 2007 to 2020. These searches were run on 7 July 2021 so that the full set of documents from 2002 to 2020 should be included. Appendix I lists the Scopus search syntax used for the four encyclopedias.

The example below is the Scopus syntax for searching the citing documents to Wikipedia in Computer Science from 2002 to 2020.

$$
\begin{aligned}
& \text { (REF(“*wikipedia.org/w*") OR REFSRCTITLE(“*wikipedia*”)) AND ((PUBYEAR > 2001) AND (PUBYEAR < 2021)) } \\
& \text { AND SUBJAREA(COMP) }
\end{aligned}
$$

The queries sometimes gave a few irrelevant matches, based on a check of 100 random citing documents to each of the four encyclopedias. The Wikipedia queries generated one false match, Baidu Baike got one while Britannica got three All the false matches were caused because the citing documents cite articles with the relevant encyclopedia names in titles. The Scholarpedia queries returned one false match ("Perception of surface stickiness in different sensory modalities: An functional MRI study") using the original syntax:

(REF(“*scholarpedia.org/article*”) OR REFSRCTITLE(scholarpedia)) AND ((PUBYEAR > 2006 ) AND (PUBYEAR < 2019))

This false citing document cites one article that was published in an encyclopedia book "Scholarpedia of Touch". We amended the syntax as below:

(REF(“*scholarpedia.org/article*”) OR REFSRCTITLE(scholarpedia) AND NOT REFSRCTITLE("scholarpedia of touch")) AND ((PUBYEAR > 2006) AND (PUBYEAR < 2021))

The new Scholarpedia search syntax did not generate any false matches in the subsequent 100 random citing documents checking. The search syntax for the other three encyclopedias remains unchanged to ensure that valid results are not excluded.

Scopus classifies articles into broad or narrow fields based on the journal in which they are published, except for general journals. This is a limitation because an article may be published within an interdisciplinary journal or an out of field journal and receive an inappropriate subject classification. This is not expected to be a substantial problem at the aggregate level reported here.

\section{Results and discussion}

Altogether Wikipedia was cited by 141,991 Scopus indexed documents (2002 to 2020), Britannica 15,929 (2002 to 2020 ), Baidu Baike 2,934 (2007 to 2020) and Scholarpedia 8,399 (2007 to 2020). The proportions of documents citing each of the four encyclopedias have increased over time, with Wikipedia being by far the most cited (Figure 1). The proportions of articles citing the crowdsourced encyclopedias, Wikipedia and Baidu Baike, have stabilized since 2012, however, with a slight decreasing trend from 2013 that seems to have accelerated in 2020. The decrease might be due to stricter editorial policies or a wider recognition of the dangers of citing unstable, editable sources. Wikipedia is now substantially more cited than the other three encyclopedias, having overtaken Britannica in 2005.

The continuing popularity of Britannica, despite its open access competitors, is partly due to citations to old editions for established procedures (e.g., the 2018 article, "Distribution of runs of homozygosity in Chinese and Western pig breeds evaluated by reduced-representation sequencing data" cites a 1948 edition for a genetic formula) and for historical references (e.g., "The Chiropractic Vertebral Subluxation Part 3: Complexity and Identity From 1908 to 1915 " cites the 1902 edition to help explain where a scientist got his knowledge from). It is presumably also useful as a relatively scholarly source of definitions or background information (e.g., the first sentence of the introduction of "Ingestion of microplastics by some commercial fishes in the lower Gulf of Thailand: A preliminary approach to ocean conservation" is "Plastic pollution is the gathering of plastic substances in the environments which have several hostile effects on wildlife, wildlife habitat as well as on human beings" with a citation to the Britannica entry on plastic pollution). Britannica references also have a more scholarly structure, half of the time including the contribution authors (which never occurs for Wikipedia), but usually also a Britannica online URL. For example, a Journal of Aesthetics and Art Criticism discussion of satire cited the Britannica article on the topic, mentioning its author, R.C. Elliot, and URL https://www.britannica.com/art/satire

Wikipedia was the most cited encyclopedia, with a maximum of $1 \%$ of Scopus documents citing it in Computer Science 


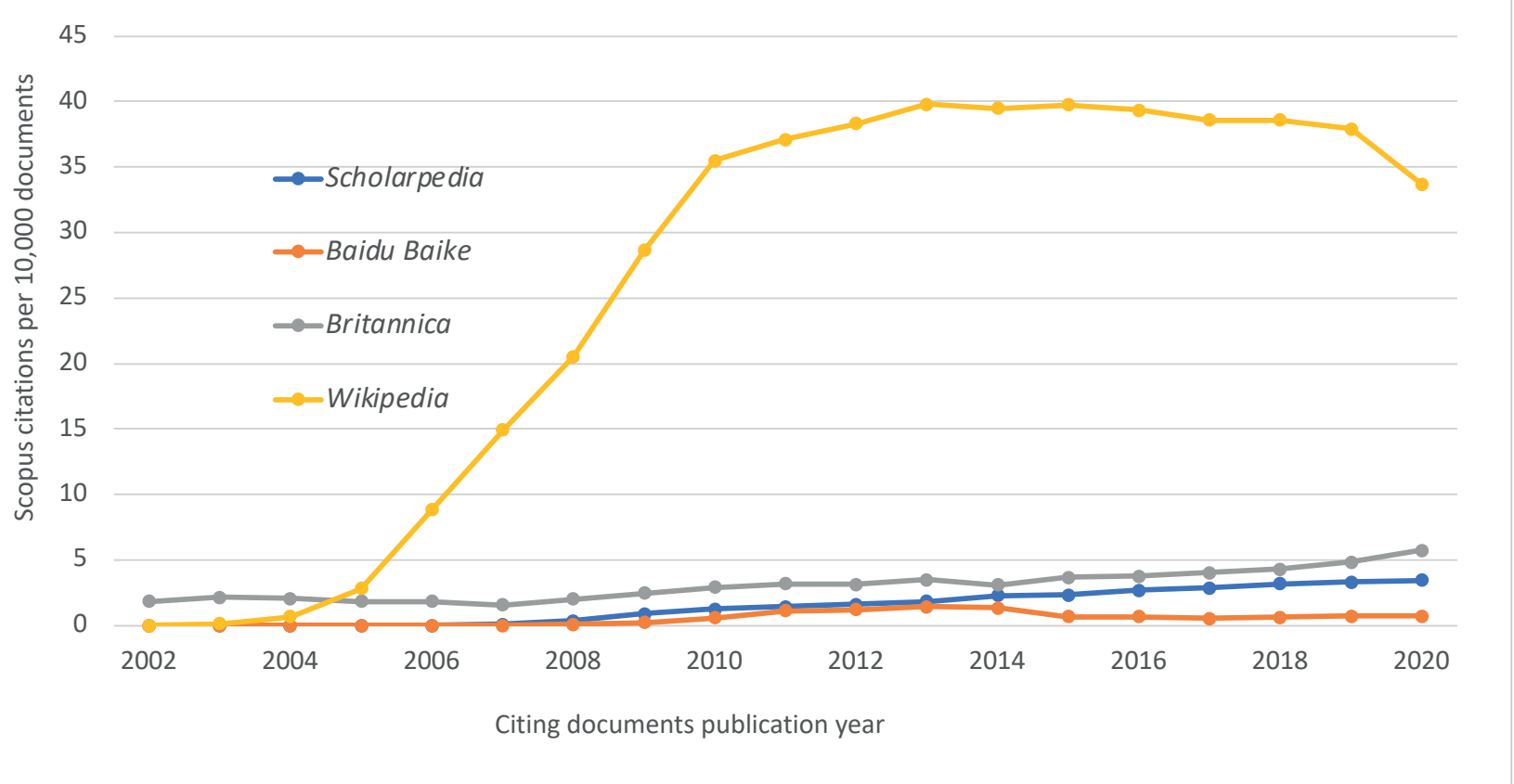

Figure 1. Scopus documents (per 10,000) citing Wikipedia, Britannica, Baidu Baike and Scholarpedia

Both expert-authored encyclopedias are increasing their relative number of citations. Whilst this is perhaps unsurprising for the relatively new Scholarpedia, the increase is unexpected for Britannica. The increase for Britannica may be partly due to reluctance to cite Wikipedia, which it may be starting to replace. It may also reflect Britannica's decision to be online only in 2012 or the success of specific sales or marketing initiatives.

\subsection{The main citing fields for each encyclopedia}

Despite the overall dominance of Wikipedia, there are substantial disciplinary differences in citing the four encyclopedias (Table 1 and Figure 2). From the uneven bar sizes for many fields and the top 10 most cited fields, it is clear that the encyclopedias have substantially different rates of use. Some notable examples are singled out here for comment.

- Scholarpedia is very highly used in Neuroscience, highly used in Mathematics and also in Physics and Astronomy. In these three areas it attracts disproportionately many citations from Scopus articles compared to the other encyclopedias (as a \% share of their cited documents). Thus, it has clear fields in which it is a highly used resource.

- Britannica is highly used in Arts and Humanities and Social Sciences, compared to the other encyclopedias. Articles in these two subject areas may reference facts or definitions for historical or cultural topics, such as a citation to a Britannica article on the Palmer raids 1919-22 in the USA.

- Baidu Baike attracts relatively many citations from Engineering and Wikipedia is not disproportionately cited in any particular field.

Some fields have disproportionately high or low encyclopedia use.

- Medicine is the main field in which all the encyclopedias are comparatively rarely cited. The related field of Biochemistry, Genetics and Molecular Biology also rarely cites encyclopedias. These areas might have fewer definitions to cite, may need more scholarly sources of citations, or may include less background information in their articles. This finding is surprising given that Wikipedia is known to have good coverage of these areas (Arroyo-Machado et al., 2020).

- Materials Science comparatively rarely cites encyclopedias, although it is not clear why.

- Computer Science has relatively many citations to Wikipedia, Baidu Baike and Scholarpedia. Presumably this is for standard definitions or background for computing terminology. For example, both Baidu Baike and Wikipedia pages on the Internet of Things (IoT) were frequently cited. The more traditional Britannica would presumably be less able to keep up with modern information technology changes. Its 2015 loT article was not cited, although its internet article from 1998 received one citation.

- Decision Sciences is a relatively heavy citer of all four encyclopedias, suggesting that this subject has a particularly strong need to cite reference works, perhaps for standard mathematical or statistical formulae or definitions. For example, the Baidu Baike article on affine transformations was cited four times.

- Social Sciences and Business, Management and Accounting are high citing areas for Wikipedia, Britannica and Baidu Baike. Articles in these two subject areas may reference facts or definitions for topics not covered well by Scholarpedia. For example, the topics of the Wikipedia pages cited included the demographics of Russia, Hurricane Maria, and Rohingya refugees in Bangladesh. 
Table 1. The top 10 subjects with the highest proportions of documents citing the four encyclopedias (per 10,000 Scopus indexed documents)

\begin{tabular}{|c|c|c|c|c|c|c|c|}
\hline Wikipedia & & Britannica & & Baidu Baike & & Scholarpedia & \\
\hline Computer Science & 100.2 & Arts and Humanities & 20.0 & Computer Science & 1.8 & Neuroscience & 14.6 \\
\hline Decision Sciences & 83.2 & Social Sciences & 12.3 & Social Sciences & 1.5 & Computer Science & 6.7 \\
\hline Social Sciences & 54.6 & Economics, Econometrics & 9.2 & Engineering & 1.5 & Mathematics & 6.7 \\
\hline $\begin{array}{l}\text { Business, Manage- } \\
\text { ment, \& Accounting }\end{array}$ & 49.2 & $\begin{array}{l}\text { Business, Management, \& } \\
\text { Accounting }\end{array}$ & 7.5 & Decision Sciences & 1.4 & Psychology & 4.8 \\
\hline Mathematics & 46.8 & Psychology & 5.1 & $\begin{array}{l}\text { Business, Management, } \\
\text { \& Accounting }\end{array}$ & 1.4 & Decision Sciences & 4.3 \\
\hline Arts and Humanities & 43.6 & Decision Sciences & 4.3 & Environmental Sciences & 1.0 & Physics and Astronomy & 4.1 \\
\hline Engineering & 43.4 & Environmental Sciences & 4.2 & Arts and Humanities & 1.0 & Multidisciplinary & 3.4 \\
\hline Energy & 39.7 & $\begin{array}{l}\text { Earth and Planetary } \\
\text { Sciences }\end{array}$ & 3.6 & Energy & 0.9 & Engineering & 2.4 \\
\hline $\begin{array}{l}\text { Economics, Econo- } \\
\text { metrics }\end{array}$ & 36.6 & $\begin{array}{l}\text { Agricultural and Biologi- } \\
\text { cal Sciences }\end{array}$ & 3.1 & $\begin{array}{l}\text { Economics, Econome- } \\
\text { trics }\end{array}$ & 0.9 & Arts and Humanities & 1.9 \\
\hline $\begin{array}{l}\text { Environmental } \\
\text { Sciences }\end{array}$ & 25.7 & Computer Science & 2.9 & Mathematics & 0.9 & $\begin{array}{l}\text { Biochemistry, Genetics and } \\
\text { Molecular Biology }\end{array}$ & 1.6 \\
\hline
\end{tabular}

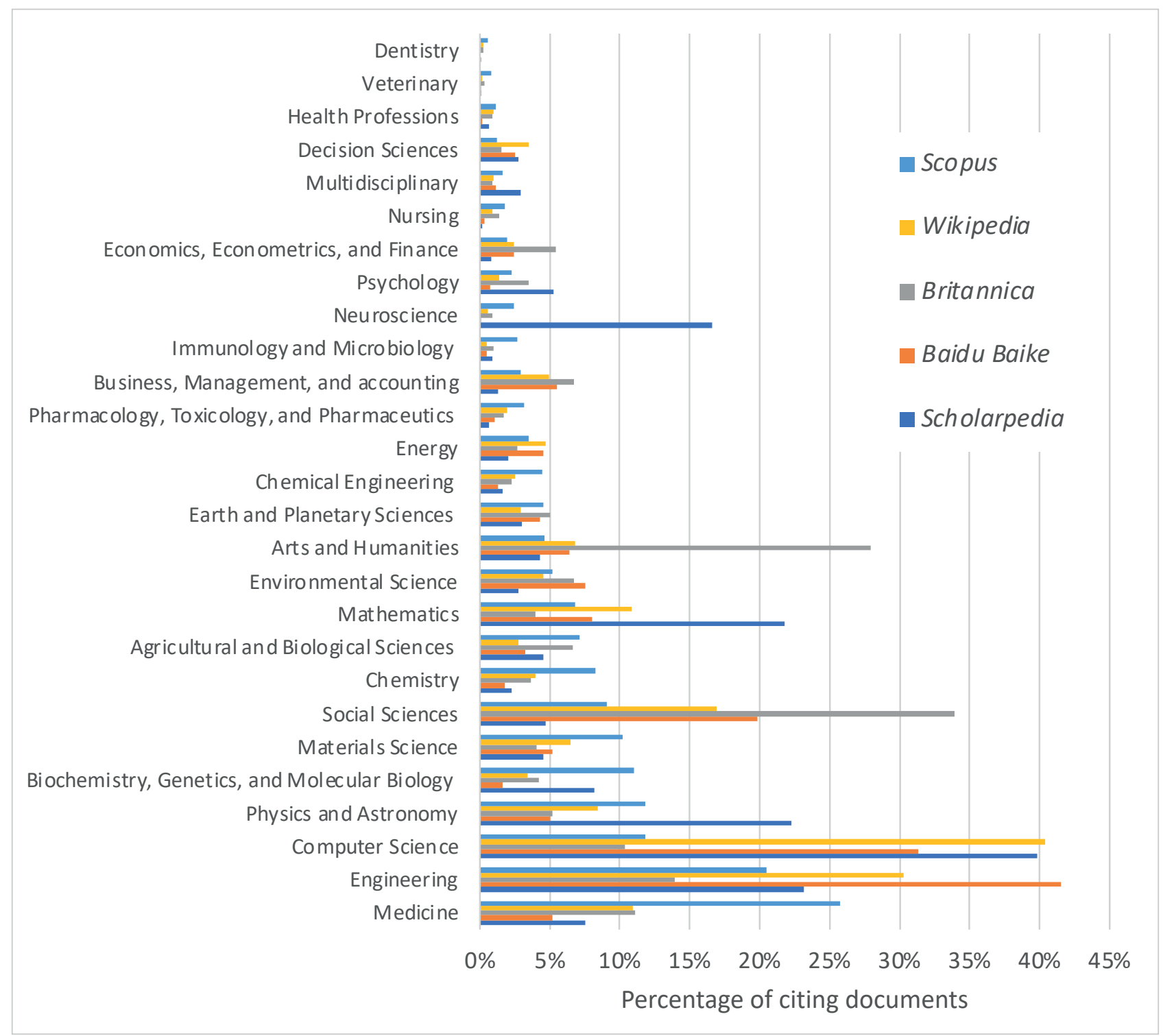

Figure 2. Scopus citations to Wikipedia, Britannica, Baidu Baike and Scholarpedia against total number of Scopus documents indexed (2002-2020). Fields $(n=27)$ are listed in ascending order of size in Scopus. The percentages are calculated out of the total data for each source. 


\subsection{Types of documents citing each encyclopedia}

Information about the types of documents citing encyclopedias may give deeper insights into who cites them and why. Open Access (OA) citing documents in Scopus are more likely to cite Scholarpedia but less likely to cite the other three encyclopedias (Figure 3), perhaps surprisingly given the open access credentials of three of them. Just under half of the citations are from Gold or Hybrid Gold journals, with the remainder from authors publishing their versions online (Green OA) or the publishers making a version of the manuscript available temporarily (Bronze OA). These figures are estimates from Scopus since articles can fit in multiple categories. For the purposes of the diagram, Green OA articles that are also Gold or Hybrid Gold or Bronze are not included in the Green figures (see Appendix II for the search syntax).

As illustrated in Figure 4, books and book series in Scopus are more likely to cite all encyclopedias. Conference papers published in proceedings are more likely than other Scopus documents to cite all encyclopedias except Britannica. Since conference papers are important in fast-moving subjects like computing, the low proportion of Britannica citations is unsurprising. Nevertheless, this suggests that journal articles (and other documents published in journals) are less likely to cite encyclopedias than other academic document types. This may reflect different attitudes of journal editors/ peer reviewers/authors towards encyclopedias or more stringent peer review for journal articles.

\subsection{Languages and countries citing each encyclopedia}

English language documents in Scopus are more likely to cite the three non-Chinese encyclopedias than are non-English documents in Scopus (Table 2). Chinese documents in Scopus are substantially more likely to cite Chinese language encyclopedia Baidu Baike. Even though Wikipedia is multilingual (alone of the four encyclopedias), it is most cited in English and with no other common language citing it much. The slight tendency for Portuguese language documents to cite English-language Britannica (nearly twice as much as Wikipedia) is an anomaly, although there are historical connections between the UK and Portugal.

Table 2. The most common 10 languages for documents citing the four encyclopedias. Bold languages are the main ones above the Scopus average

\begin{tabular}{|l|c|l|r|l|r|l|c|l|c|}
\hline \multicolumn{1}{|c|}{ Scopus } & $\%$ & Wikipedia & $\%$ & Britannica & $\%$ & Baidu Baike & $\%$ & Scholarpedia & $\%$ \\
\hline English & 90.8 & English & 95.4 & English & 95.9 & English & 88.4 & English & 98.5 \\
\hline Chinese & 3.5 & German & 1.1 & Spanish & 1.0 & Chinese & 11.1 & Chinese & 0.8 \\
\hline German & 1.3 & Spanish & 0.8 & Portuguese & 0.5 & German & 0.1 & Russian & 0.2 \\
\hline French & 1.1 & Chinese & 0.7 & German & 0.5 & Russian & 0.1 & Spanish & 0.1 \\
\hline Spanish & 1.0 & French & 0.5 & French & 0.4 & French & 0.1 & Turkish & 0.1 \\
\hline Russian & 0.6 & Russian & 0.3 & Croatian & 0.4 & Spanish & 0.1 & German & 0.1 \\
\hline Japanese & 0.6 & Portuguese & 0.3 & Russian & 0.3 & Bulgarian & 0.0 & Portuguese & 0.1 \\
\hline Portuguese & 0.5 & Turkish & 0.2 & Italian & 0.2 & Italian & 0.0 & French & 0.1 \\
\hline Italian & 0.3 & Polish & 0.2 & Slovenian & 0.2 & Korean & 0.0 & Italian & 0.0 \\
\hline Polish & 0.2 & Croatian & 0.2 & Chinese & 0.2 & Polish & 0.0 & Japanese & 0.0 \\
\hline
\end{tabular}

Documents from the USA are more likely to cite Wikipedia and Britannica (Table 3). China dominates Baidu Baike citations, presumably because of its language, and other Chinese-speaking countries/territories also disproportionately cite it. Scholarpedia citations are disproportionately from Germany, despite its origins in the USA. This may be a topic issue, if Neuroscience is a German specialty. 
Surprisingly, the UK does not cite Wikipedia disproportionately often. India, South Korea, Australia and Taiwan testify to the international credibility of Wikipedia, although all four countries presumably publish most academic research in English (indexed in Scopus) and have historical connections to the UK or USA. Britannica, originally from the UK but published in the USA since 1901 (Encyclopædia Britannica, 2019), is relatively highly cited only in countries with a historical connection to the UK.

Table 3. The most common 10 countries/territories for documents citing the four encyclopedias. Countries/territories are bold when they are the main country above the Scopus average

\begin{tabular}{|c|c|c|c|c|c|c|c|c|c|}
\hline Scopus & $\%$ & Wikipedia & $\%$ & Britannica & $\%$ & Baidu Baike & $\%$ & Scholarpedia & $\%$ \\
\hline USA & 23.9 & USA & 25.5 & USA & 29.1 & China & 86.9 & USA & 26.4 \\
\hline China & 14.7 & China & 12.1 & UK & 9.6 & USA & 8.8 & China & 13.1 \\
\hline UK & 7.1 & India & 10.9 & India & 4.7 & Hong Kong & 2.4 & Germany & 11.3 \\
\hline Germany & 6.1 & UK & 5.7 & Canada & 4.6 & Australia & 2.3 & UK & 10.6 \\
\hline Japan & 5.1 & Germany & 4.0 & Germany & 4.4 & UK & 2.3 & France & 7.5 \\
\hline France & 4.2 & South Korea & 3.7 & Australia & 3.7 & Canada & 1.4 & Italy & 5.5 \\
\hline India & 4.1 & Canada & 3.4 & China & 3.0 & Taiwan & 1.3 & India & 5.2 \\
\hline Italy & 3.7 & Australia & 3.1 & Italy & 2.7 & Singapore & 0.7 & Canada & 4.4 \\
\hline Canada & 3.6 & Taiwan & 2.4 & France & 2.1 & South Korea & 0.6 & Japan & 3.8 \\
\hline Australia & 3 & Japan & 2.2 & Spain & 1.9 & Japan & 0.6 & Spain & 3.7 \\
\hline
\end{tabular}

\section{Limitations}

This study relies on Scopus advanced search facilities for data gathering. As a result, it is limited by Scopus's coverage, classification schemes and search efficiencies. For example, Scopus covers more publications than does the Web of Science, but it does not cover all scholarly publications, and it may miss book chapters and Chinese

Peer-reviewed encyclopedia Britannica citations continue to be valuable in the Arts and Humanities, and Scholarpedia citations in Neuroscience journals which are important sources that cite encyclopedias. This may explain why Scopus citations to Baidu Baike are much fewer than those counting from Chinese article index databases (Ding et al., 2013; Wang, 2016).

Although in collaboration with Impactstory, Scopus may miss open access documents in its search results as it only sourced open access documents that are harvested by Unpaywall -a database run by Impactstory (Scopus: Access and use Support Center, 2021).

Finally, it is important to be cautious when interpreting the results of citing patterns by subjects because Scopus does not index all scholarly publications and its comprehensiveness varies between fields.

\section{Conclusions}

Citing encyclopedias is rare in all academic subjects. Although Wikipedia is the most cited encyclopedia, according to Scopus data, in the 27 subjects investigated it occurred in a maximum of $1 \%$ of Scopus documents citing it in Computer Science.

In answer to Question 1:

For the two free publicly editable encyclopedias: citations to Wikipedia increased exponentially until 2010, when the rate of increase slowed down and then started to decrease in 2020 (updating a previous study showing citations continuing to increase: Tomaszewski \& MacDonald, 2018) while citations to Baidu Baike decreased substantially after 2013.

For the two peer-reviewed encyclopedias: citations to Britannica relatively stabilized over the years while citations to Scholarpedia increasing gradually since it started in 2006. Thus, with the possible exception of Neuroscience, citations to major encyclopedias should continue to be rare in the future and librarians/authors/reviewers should not expect to see or use them other than in exceptional circumstances.

They may also see a partial reversion from crowdsourced encyclopedias to expert-written versions. In the context of apparently increasing public scrutiny of academic research (e.g., during the Covid-19 pandemic), it seems particularly important to ensure that all citations are robust. In this context, a citation to a crowdsourced encyclopedia may be a weak point in an article that may be exploited, particularly if the article covers a controversial topic.

In answer to Question 2:

There are substantial disciplinary differences in the uptake of the four encyclopedias, and they have particularly little value in Medicine. The four encyclopedias seem to be particularly useful in mathematical areas, such as

Unsurprisingly, Baidu Baike is disproportionately cited by Chinese-speaking countries/territories 
Decision Sciences, presumably for definitions, and in fast moving technological areas, such as Computing, presumably also for definitions as well as explanations of new technological developments. In contrast, Britannica citations continue to be valuable in the Social Sciences and Arts and Humanities. Its relative lack of citations in other areas may reflect its targeting of a general audience for which detailed scientific explanations and coverage would be inappropriate and not cost-effective to curate. This seems like a niche that crowdsourced encyclopedias will continue to fill. Scholarpedia provides a partial exception to this, by providing detailed peer reviewed expert articles on highly scientific topics, although its coverage is limited and it is not clear if the model is sustainable across academia. Scholars and librarians that (occasionally) need to cite encyclopedias may therefore consider first checking the one most used in their fields.

In answer to Question 3:

Books and book series in Scopus are more likely to cite the four encyclopedias than for general Scopus-indexed documents. This may be due to less strict refereeing for books or more encyclopedia-like content in books (e.g., handbooks). Open access citing documents are more likely to cite Scholarpedia but less likely to cite the other three counterparts. This updates a previous study of Wikipedia that did not find OA publications to be the main sources of scholarly citations to Wikipedia (Tomaszewski; MacDonald, 2018). Chinese documents are more likely to cite Baidu Baike while English documents are more likely to cite the other three non-Chinese encyclopedias. Unsurprising, Baidu Baike is highly cited by Chinese-speaking countries/territories, US documents are more likely to cite the three non-Chinese encyclopedias while Scholarpedia is more cited by nearly all the major publishing nations except China and Japan. Thus, the rate of citing encyclopedias varies between countries and document types, suggesting that scholars choose sources that are known to them rather than selecting the best source for any particular citation.

In summary, encyclopedias are continuing to play a minor role in formal scholarly communication, in the form of references. National factors play a role, with authors being more likely to select encyclopedias that are popular in their countries. Despite the criticism of the open editing formats of Wikipedia and Baidu Baike, each of the four major encyclopedias investigated seems to have found a niche. It is not clear whether the open encyclopedias are cited by scholars that are aware of and accept their limitations, however, or whether in the case of the two unrefereed sources, there are mistaken attempts to underpin research with unstable sources. This is an important issue for the scholarly community, and one that editors and reviewers should monitor.

\section{Open-access citing documents in Scopus are more likely to cite Scholarpedia but less likely to cite the other three ency- clopedias}

Encyclopedias have minor value for academic research, often for background and definition purposes

\section{References}

Arroyo-Machado, Wenceslao; Torres-Salinas, Daniel; Herrera-Viedma, Enrique; Romero-Frías, Esteban (2020). "Science through Wikipedia: A novel representation of open knowledge through co-citation networks". PloS one, v. 15, n. 2 , e0228713.

https://doi.org/10.1371/journal.pone.0228713

Baker, Daniel J. (2011). "A Jester's promenade: Citations to Wikipedia in law reviews, 2002-2008". Journal of law and policy for the information society, v. 7, n. 2, pp. 361-404.

https://doi.org/10.2139/ssrn.1525619

Bould, M. Dylan; Hladkowicz, Emily S.; Pigford, Ashlee-Ann E.; Ufholz, Lee-Anne; Postonogova, Tatyana; Shin, Eunkyung; Boet, Sylvain (2014). "References that anyone can edit: Review of Wikipedia citations in peer reviewed health science literature". BMJ, v. 348, g1585.

https://doi.org/10.1136/bmj.g1585

Brazzeal, Bradley (2011). "Citations to Wikipedia in chemistry journals: A preliminary study". Issues in science and technology librarianship, v. Fall. https://doi.org/10.5062/F4057CV7

Chesney, Thomas (2006). "An empirical examination of Wikipedia's credibility". First Monday, v. 11, n. 11. https://doi.org/10.5210/fm.v11i11.1413

Chinese Social Sciences Citation Index (2015). Wikipedia.

https://en.wikipedia.org/w/index.php?title=Chinese_Social_Sciences_Citation_Index\&oldid=682574308

CNKI (2019). Wikipedia.

https://en.wikipedia.org/w/index.php?title=CNKI\&oldid=913654910

Colavizza, Giovanni (2020). “Covid-19 research in Wikipedia”. Quantitative science studies, v. 1, n. 4, pp. 1349-1380. https://doi.org/10.1162/qss_a_00080 
Ding, Yudong; Zhang, Chunfeng; Liu, Ying (2013). "Statistics and analysis of wiki-based Chinese online-encyclopedia cited by journal papers". Journal of intelligence, v. 32, n. 3. http://en.cnki.com.cn/Article_en/CJFDTOTAL-QBZZ201303019.htm

Encyclopædia Britannica (2019). Wikipedia.

https://en.wikipedia.org/w/index.php?title=Encyclop\%C3\%A6dia_Britannica\&oldid=931322238

Fallis, Don (2008). "Toward an epistemology of Wikipedia". Journal of the American Society for Information Science and Technology, v. 59, n. 10, pp. 1662-1674.

https://doi.org/10.1002/asi.20870

Giles, Jim (2005). "Internet encyclopaedias go head to head". Nature, v. 438, pp. 900-901. https://doi.org/10.1038/438900a

Holman-Rector, Lucy (2008). "Comparison of Wikipedia and other encyclopedias for accuracy, breadth, and depth in historical articles". Reference services review, v. 36, n. 1, pp. 7-22.

https://doi.org/10.1108/00907320810851998

Huggett, Sarah (2012). "The influence of free encyclopedias on science". Research trends, n. 27. https://www.researchtrends.com/issue-27-march-2012/the-influence-of-free-encyclopedias-on-science

Izhikevich, Eugene (2006). "Scholarpedia". Scholarpedia, v. 1, n. 2, pp. 1.

https://doi.org/10.4249/scholarpedia.1

Jemielniak, Dariusz; Masukume, Gwinyai; Wilamowski, Maciej (2019). "The most influential medical journals according to Wikipedia: Quantitative analysis". Journal of medical internet research, v. 21, n. 1, e11429.

https://doi.org/10.2196/11429

Jullien, Nicolas (2012). "What we know about Wikipedia: A review of the literature analyzing the project (s)". SSRN, 86 pp. https://doi.org/10.2139/ssrn.2053597

Kousha, Kayvan; Thelwall, Mike (2017). "Are Wikipedia citations important evidence of the impact of scholarly articles and books?". Journal of the Association for Information Science and Technology, v. 68, n. 3. pp. 762-779.

https://doi.org/10.1002/asi.23694

Kousha, Kayvan; Thelwall, Mike; Abdoli, Mahshid (2012). "The role of online videos in research communication: A content analysis of YouTube videos cited in academic publications". Journal of the American Society for Information Science and Technology, v. 63, n. 9, pp. 1710-1727.

https://doi.org/10.1002/asi.22717

Li, Xuemei; Thelwall, Mike; Kousha, Kayvan (2015). "The role of arXiv, RePEc, SSRN and PMC in formal scholarly communication". Aslib journal of information management, v. 67, n. 6, pp. 614-635. https://doi.org/10.1108/AJIM-03-2015-0049

Lih, Andrew (2004). "Wikipedia as participatory journalism: Reliable sources? Metrics for evaluating collaborative media as a news resource". In: $5^{\text {th }}$ International symposium on online journalism.

http://www.ufrgs.br/limc/participativo/pdf/wikipedia.pdf

Lin, Jennifer; Fenner, Martin (2014). "An analysis of Wikipedia references across PLOS publications". In: Altmetrics14: Expanding impacts and metrics an ACM web science conference 2014 workshop, pp. 23-26.

https://figshare.com/articles/journal_contribution/An_analysis_of_Wikipedia_references_across_PLOS_ publications/1048991

Mesgari, Mastafa; Okoli, Chitu; Mehdi, Mohamad; Nielsen, Finn-Arup; Lanamäki, Arto (2015). "'The sum of all human knowledge': A systematic review of scholarly research on the content of Wikipedia". Journal of the Association for Information Science and Technology, v. 66, n. 2, pp. 219-245.

https://doi.org/10.1002/asi.23172

Messner, Marcus; DiStaso, Marcia W. (2013). "Wikipedia versus Encyclopedia Britannica: A longitudinal analysis to identify the impact of social media on the standards of knowledge". Mass communication and society, v. 16, n. 4, pp. 465-486. https://doi.org/10.1080/15205436.2012.732649

Okoli, Chitu; Mehdi, Mohamad; Mesgari, Mastafa; Nielsen, Finn-Arup; Lanamäki, Arto (2012). "The people's encyclopedia under the gaze of the sages: A systematic review of scholarly research on Wikipedia". SSRN. https://doi.org/10.2139/ssrn.2021326

Okoli, Chitu; Mehdi, Mohamad; Mesgari, Mastafa; Nielsen, Finn-Arup; Lanamäki, Arto (2014). "Wikipedia in the eyes of its beholders: A systematic review of scholarly research on Wikipedia readers and readership". Journal of the Association for Information Science and Technology, v. 65, n. 12, pp. 2381-2403.

https://doi.org/10.1002/asi.23162 
Park, Taemin-Kim (2011). "The visibility of Wikipedia in scholarly publications". First Monday, v. 16, n. 8. https://doi.org/10.5210/fm.v16i8.3492

Priem, Jason; Piwowar, Heather A.; Hemminger, Bradley M. (2012). "Altmetrics in the wild: Using social media to explore scholarly impact". ArXiv.

http://arxiv.org/abs/1203.4745

Samoilenko, Anna; Yasseri, Taha (2014). "The distorted mirror of Wikipedia: a quantitative analysis of Wikipedia coverage of academics". EPJ data science, v. 3, n. 1.

https://doi.org/10.1140/epjds20

Scopus (2021). How do I find Open Access journals and articles in Scopus?. Scopus: Access and use Support Center. https://service.elsevier.com/app/answers/detail/a_id/11268/supporthub/scopus/kw/open+access

Shoyama, Rex (2014). "Citations to Wikipedia in Canadian law journal and law review articles". Canadian law library review, v. 39, n. 12, pp. 11-15.

https://ssrn.com/abstract $=2578678$

Tohidinasab, Fariba; Jamali, Hamid R (2013). "Why and where Wikipedia is cited in journal articles?". Journal of scientometric research, v. 2, n. 3, pp. 231-238.

https://doi.org/10.4103/2320-0057.135415

Tomaszewski, Robert (2018). "A comparative study of citations to chemical encyclopedias in scholarly articles: Kirk-Othmer Encyclopedia of Chemical Technology and Ullmann's Encyclopedia of Industrial Chemistry". Scientometrics, v. 117, n. 1 , pp. 175-189.

https://doi.org/10.1007/s11192-018-2844-1

Tomaszewski, Robert; MacDonald, Karen I. (2018). "A study of citations to Wikipedia in scholarly publications". Science \& technology libraries, n. 35, n. 3, pp. 246-261.

https://doi.org/10.1080/0194262X.2016.1206052

Wang, Zhihong (2016). "Research on characteristics of online encyclopedia cited by LIS journal articles in China". Library and information service, v. 60, n. 19, pp. 99-107.

http://en.cnki.com.cn/Article_en/CJFDTotal-TSQB201619016.htm

\section{Appendixes}

\section{Appendix I}

\section{Scopus syntax for searching citing documents to the four encyclopedias}

Query for documents citing Wikipedia (2002 to 2020):

(REF(“*wikipedia.org/w*”) OR REFSRCTITLE(“*wikipedia*”)) AND ((PUBYEAR > 2001) AND (PUBYEAR < 2021))

Query for documents citing Britannica (2002 to 2020):

(REF(“*britannica.com*”) OR REFSRCTITLE((“*Encyclopædia Britannica*” OR “*Encyclopaedia Britannica*” OR

“*Encyclopedia Britannica*” OR “*Britannica Online*”))) AND ((PUBYEAR > 2001) AND (PUBYEAR < 2021))

Query for documents citing Baidu Baike (2007 to 2020):

(REF(“*baike.baidu.com*”) OR REFSRCTITLE(“baidu baike” OR “百度百科”)) AND ((PUBYEAR > 2006) AND (PUBYEAR < 2021))

Query for documents citing Scholarpedia (2007 to 2020):

(REF("*scholarpedia.org/article*") OR REFSRCTITLE(scholarpedia) AND NOT REFSRCTITLE("scholarpedia of touch")) AND ((PUBYEAR > 2006) AND (PUBYEAR < 2021))

\section{Appendix II}

Scopus syntax for searching Green OA documents that are not Gold, Hybrid Gold or Bronze

OA(repository) AND NOT (OA(publisherfullgold) OR OA(publisherhybridgold) OR OA(publisherfree2read) 